\title{
FOUR DIMENSIONAL TOPOLOGICAL QUANTUM FIELD THEORY, HOPF CATEGORIES, AND THE CANONICAL BASES
}

by Louis Crane, Department of Mathematics, KSU

and Igor B. Frenkel, Department of Mathematics, Yale University

Abstract: We propose a new combinatorial method of constructing 4D-TQFTs. The method uses a new type of algebraic structure called a Hopf category. We also outline the construction of a family of Hopf categories related to the quantum groups, using the canonical bases.

\section{INTRODUCTION}

Since the seminal papers of Witten [1], and Atiyah [2], the attention of many mathematicians and physicists has been drawn to the problem of constructing topological quantum field theories (TQFTs). The mathematical form of the definition of a TQFT uses very categorical language, and is strongly influenced by the definition of a conformal field theory due to Segal [3]. The subject of TQFT has a dual character, as a new development in algebraic topology on one hand, and as a branch of quantum field theory on the other. While physicists have suggested that various TQFTs exist in various dimensions by non-rigorous and ill-defined arguments involving path integrals, many rigorous constructions have been discovered using various techniques involving abstract algebra $[4,5,6,7,8,9]$. There has been a close interaction between the mathematical and physical approaches, which has led to the rapid development of the subject.

The most exciting theories whose existence has been suggested are the cousins of Donaldson-Floer (DF) theory [1], which should exist in 4 dimensions, and be sensitive to smooth structure. So far, neither the analytic [10] nor the path integral nor any algebraic approach has succeeded in constructing Donaldson-Floer theory, except for special cases.

The only 4D-TQFTs which have been rigorously constructed so far are Dijkgraaf-Witten theory $[11,12,13]$, which counts representations of $\pi_{1}$ into a finite group, and Crane-Yetter theory [14], which can be used to find the signature, Euler character, second Chern class and cup product on the second cohomology of the 4 manifold. Both of these theories, in short, produce classical invariants (although by new combinatorial formulae), and hence, are not obviously related to Donaldson-Floer theory.

The purpose of this paper is to propose a new algebraic construction of a $4 \mathrm{D}-\mathrm{TQFT}$, which uses a novel algebraic structure, called a Hopf category. This would not be very interesting, except that we also propose a construction of a family of Hopf categories, intimately related to the canonical bases for the quantum groups. We conjecture that the 4D-TQFTs which arise by our new method from our Hopf categories may be sensitive to smooth structure, and in fact may be related to DF theory.

As far as the current status of DF theory is concerned, let us make a hypothetical historical analogy. Suppose somebody had used the implicit function theorem to show that the dimension of the space of harmonic forms on a Riemannian manifold was independent of the metric. One would then have a set of "analytic" invariants of closed manifolds. It would then take some imagining to find a combinatorial algebraic formula for these invariants from a triangulation. Of course, once that invariant had been understood as rational cohomology, nobody would think of de Rham's definition of it as truly fundamental any more.

DF invariants come from counting instantons, a sort of nonabelian version of harmonic forms. It would not be too surprising if the combinatorial version of these invariants utilized noncommutative algebra.

Let us try to explain some of the ideas which underly our construction.

The first is that the constructions of particular types of TQFTs in various dimensions, written in terms of generators and relations, end up requiring precisely a family of classical structures of abstract algebra. Let us give just one simple example to illustrate this. If we try to construct a 2D-TQFT from triangulated surfaces by putting labels i on edges and attaching numbers $C_{i j k}$ depending on the edge labels to triangles, then the topological move:

Figure 1

tells us that we need $C_{i j k}$ to be the structure constants of an associative algebra (in fact, it tells us a lot more, namely, that we need a symmetric Frobenius algebra). Schematically, we can draw the associative law as follows:

Figure 2

The other topological moves on triangulations tell us that we actually need an associative algebra with a rigid structure of a classically familiar type, namely a semisimple algebra [9]. 
The second idea motivating our construction is that replacing an algebraic structure with a similar categorical algebraic structure lifts the dimension of the corresponding TQFT by 1 . For example, we can construct a 3D-TQFT from a suitable tensor category by analogy with the construction discussed above. The $3 \mathrm{D}$ analog of the move illustrated above is the famous Stasheff pentagon [15], also known as the BiedenharnEliot relation [16]. This is essentially the construction in [8].

The third idea leading up to our construction is that it is possible to use a Hopf algebra to construct a $3 \mathrm{D}-\mathrm{TQFT}$, by a process analogous to the use of an algebra in 2D, combining the use of the algebra structure on the 2-skeleton with the coproduct structure on the dual 2-skeleton of a 3D triangulation [7].

The combination of the last two ideas might suggest that a categorical version of a Hopf algebra might pull us up to $4 \mathrm{D}-\mathrm{TQFT}$. We call the new algebraic structures, which can be viewed as generators and relations for 4D-TQFT, Hopf categories.

To proceed any farther we need a miracle, namely, the existence of an interesting family of Hopf categories.

The next important input is the existence of the canonical bases [17] for a special family of Hopf algebras, namely the quantum groups. These bases are actually an indication of the existence of a family of Hopf categories, with structures closely related to the quantum groups. In fact, the structure coefficients of the quantum groups in the canonical bases are positive integers, which can be replaced by vector spaces. In this paper, we actually construct the Hopf category in the simplest case, the one corresponding to sl(2), directly.

Our construction of a 4D-TQFT is by no means complete. However, we do have at least an outline of all the major steps.

The structure of this paper is as follows: In Chapter 1 we recall the main ideas of the structure of Hopf algebras, and explain how they translate into 3D-TQFT. Chapter 2 gives the definition and essential properties of a Hopf category. Chapter 3 contains our new formula, which we call the "tornado," for constructing a 4D-TQFT from a Hopf category. In Chapter 4 we construct a non-trivial example of a Hopf category from the quantum group $U_{q} s l(2)$ using the canonical bases. Finally in Chapter 5 we discuss mathematical and physical implications. 


\section{CHAPTER 1. THE STRUCTURE OF HOPF ALGEBRAS AND 3D TQFT}

The structure of finite dimensional complex Hopf algebras in spite of its seeming simplicity is rather intricate and has only been gradually understood during the last three decades. It turns out that many known facts and formulas can be elegantly expressed via three dimensional topological quantum field theory. We will only recall those facts and formulas that enter into the topological construction.

Let $A$ be a complex finite-dimensional associative algebra with a multiplication $M$ and unit $e$, and let $A$ also be an associative coalgebra (i.e. $A^{*}$ an associative algebra) with the comultiplication $\Delta$ and counit $\varepsilon$. Then $A$ is a bialgebra if the pairs $(M, e)$ and $(\Delta, \varepsilon)$ satisfy natural consistency relations:

$$
\Delta(x y)=\Delta(x) \Delta(y), \quad \Delta e=e \otimes e, \varepsilon(x y) \quad=\varepsilon(x) \varepsilon(y), \quad \varepsilon(e)=1
$$

where $x, y \in A$. Clearly, if $A$ is a bialgebra, then $A^{*}$ is a bialgebra and vice versa.

Any element $\lambda \in A^{*}$ defines an associative bilinear form $(x, y)_{\lambda}=\lambda(x y), x, y \in A$. If there exists a nonsingular $\lambda \in A^{*}$, i.e. one where the corresponding form is nondegenerate, then the algebra $A$ is called Frobenius. Now let $A$ be a bialgebra and let $\lambda \in A^{*}$ also define a structure of a Frobenius algebra. If we require a natural consistency condition

$$
\chi \lambda=\chi(e) \lambda, \quad \chi \in A^{*}
$$

then we arrive at the notion of a Hopf algebra. The element $\lambda$ is called a left integral. In fact, a result of Larson and Sweedler [18] asserts that the existence of a nonsingular left integral $\lambda$ in a bialgebra $A$ is equivalent to the existence of the antipode $s$. As a corollary, the existence of such a $\lambda$ implies the existence of a nonsingular left integral $\ell \in A$. Thus a Hopf algebra can be viewed as a Frobenius bialgebra with certain natural consistency relations.

The above discussion remains true if we replace a left integral $\lambda \in A^{*}$ by a right integral $\rho \in A^{*}$ satisfying

$$
\rho \chi=\chi(e) \rho, \quad \chi \in A^{*} .
$$

We also denote by $r$ a right integral in $A$.If all four integrals exist they are unique up to a scalar factor.

Let us define the left and right action of $A$ on $A^{*}$ by the formulas

$$
(x-\chi)(y)=\chi(y x), \quad(\chi<x)(y)=\chi(x y)
$$

where $x, y \in A$ and $\chi \in A^{*}$. We have the following expressions of the antipode and its inverse in terms of the integrals

$$
\begin{aligned}
s(x) & =\frac{1}{\lambda(\ell)}(\lambda \leftarrow x) \rightarrow \ell=\frac{1}{\rho(r)} r<(x \rightarrow \rho) \\
s^{-1}(x) & =\frac{1}{\lambda(r)}(x \rightarrow \lambda) \rightarrow r=\frac{1}{\rho(e)} \ell \leftarrow(\rho \leftarrow x)
\end{aligned}
$$

Note that the normalization factors are always nonzero numbers. Next we introduce a special group-like element $a \in A$, i.e. $\Delta a=a \otimes a, s(a)=a^{-1}$, by any of the two equivalent conditions

$$
\lambda \chi=\chi(a) \lambda, \quad \chi \rho=\chi\left(a^{-1}\right) \rho, \quad \chi \in A^{*} .
$$

Using the duality between $A$ and $A^{*}$ we also define a second group-like element $\alpha \in A^{*}$. These two group-like elements and the number $\alpha(a)$, which turns out to be a root of unity, play an important structural role. In particular, one has

$$
\begin{aligned}
s(\lambda) & =a-\lambda & s(\rho) & =\rho\left\llcorner a^{-1},\right. \\
s^{2}(\lambda) & =\alpha(a) \lambda, & s^{2}(\rho) & =\alpha(a) \rho
\end{aligned}
$$

One of the central structural facts about Hopf algebras is the Radford formula [19]

$$
s^{4}(x)=a^{-1}\left(\alpha \rightarrow x<\alpha^{-1}\right) a, \quad x \in A .
$$


Kuperberg [20] defined an important special subclass of balanced Hopf algebras for which one has

$$
s^{2}(x)=a^{-1} x a=\alpha \rightarrow x<\alpha^{-1}, \quad x \in A .
$$

This subclass is important for the following two reasons: first "halves" of quantum groups are balanced Hopf algebras, second balanced Hopf algebras admit an elegant topological interpretation. The relation between these two facts leads to a combinatorial construction of topological quantum field theories in $3 D$, which we believe will be closely related to CSW theory.

An important property of balanced Hopf algebras is a quasisymmetry of the forms defined by left and right integrals

$$
\lambda(x y)=\lambda\left(y\left(x<\alpha^{-1}\right)\right), \quad \rho(x y)=((\alpha-y) x) .
$$

For general Hopf algebras, the analogue of the quasisymmetry relation has a more complicated form.

All the above formulas simplify significantly in the involutive case $s^{2}=i d$. It was shown by Larson and Radford [21] that this condition is equivalent to the semisimplicity of $A$, and therefore of $A^{*}$.

In this case the group-like elements $a$ and $\alpha$ coincide with units in $A$ and $A^{*}$, respectively. Thus left and right integrals coincide and they can be normalized in such a way that

$$
\lambda^{2}=\lambda, \quad \ell^{2}=\ell, \quad \lambda(\ell)=(\operatorname{dim} A)^{-1} .
$$

As we will explain below, the semisimple case admits an especially simple topological realization.

\section{Three Dimensional TQFT and Hopf Algebras}

The labyrinth of identities we have just reviewed, at least in the case of balanced (and especially semisimple) Hopf algebras, can be visualized by means of three dimensional topology. One could also say that the combinatorics of three dimensional triangulations naturally leads to the identities for Hopf algebras. This approach to constructing a $3 \mathrm{D}-\mathrm{TQFT}$ is originally due to Kuperberg $[7,20]$. Kuperberg's work is very important, although he did not quite finish the non-involutory case, since he did not find a good normalization, and could not control a projective factor of a root of unity. We sketch a slightly modified version of it here.

The main idea is to begin with a triangulation of a 3-manifold. (Actually, any polyhedral decomposition will do as well.) One labels the edges of the faces (combinatorial 1-2 flags) with elements of a basis for the algebra, combines around faces with the multiplication, and around edges with the comultiplication, and sums over labellings. Then the requirement of topological invariance will encode the identities of a Hopf algebra.

In order to absorb various powers of group like elements $a$ and $\alpha$ we define an infinite sequence of integrals by

$$
\mu^{n-\frac{1}{2}}=a^{n} \rightarrow \lambda, \quad n \in \mathbb{Z} .
$$

In fact, since the order of $a$ and $\alpha$ is finite, we get a cyclic family. By definition $\mu^{-\frac{1}{2}}$ is a left integral and $\mu^{\frac{1}{2}}$ is a right integral.

In order to actually define an invariant associated to a triangulated or polyhedrally decomposed 3manifold from a balanced Hopf algebra, we need to associate some decorations to the decomposition. Specifically, we attach an integer to each edge, a half-integer and an orientation to each face, and a preferred vertex (an "origin") to each face as well.

Figure 3

Definition Let A be a finite dimensional Hopf algebra, B a basis for it, and let (M, T) be a triangulated 3-manifold. A coloring of $(\mathrm{M}, \mathrm{T})$ by $\mathrm{A}$ in the basis $\mathrm{B}$ is a function which assigns to each pair of one face and one incident edge in $(\mathrm{M}, \mathrm{T})$ a basis element. we denote the set of colorings of (M,T) by $\mathrm{A}$ as $\Lambda(M, T, A, B)$. We will frequently suppress the mention of the basis.

Let us denote a coloring of the edges of one face by the elements of the basis of $A: x_{i_{1}}, \ldots, x_{i_{n}}$. Then to this polygon $\mathrm{P}$ (as in Figure 3) and to each coloring $\lambda \in \Lambda$ we attach the following number

$$
\#(P, \lambda)=\mu^{r}\left(s^{k_{1}}\left(x_{i_{1}}\right) \ldots s^{k_{n}}\left(x_{i_{n}}\right)\right)
$$

The antiautomorphism property of the antipode guarantees that the simultaneous shift $k_{j} \rightarrow k_{j}+1$ together with a change of orientation and the sign of $r$ changes this number by a factor of $\alpha(a)$. Clearly 
repeated application of this shift preserves the orientation and the sign of $r$.In the case of a semisimple Hopf algebra, the decoration of the polygon can be simplified: namely, one does not need to specify a special vertex and a semiintegral number $r$, also the integers attached to edges are defined mod 2 .

Since we are coloring combinations of edges incident to faces, we have a loop of labels around each edge. We can think of these as lying on the edges of a dual polygon, ie a polygon in the dual decomposition to T. We now form a number for each dual polygon and each labelling. The formula we use for $\#(E, \lambda)$ is dual to the formula above, i.e. we use the multiplication and left integral on $A^{*}$.

To form an invariant of a triangulated 3-dimensional manifold $(M, T)$, we form a sum

$$
\sum_{\lambda \in \Lambda} \prod_{\text {faces }} \#(P, \lambda) \prod_{\text {edges }} \#(E, \lambda),
$$

where we have the following restriction on decorations. If a polygon intersects a dual polygon then the difference of the integers attached to the edge of intersection should be even or odd depending whether the orientations of the polygon and the dual polygon agree or not.

In the semisimple case, this gives a TQFT. In the more general balanced case, we need some further restrictions on decorations. Namely, for any closed contractible contour on the surface obtained as a regular neighborhood of the 1-skeleton we have a certain linear relation on the numbers attached to the edges and faces of polygons. The fact that these restrictions admit solutions follows from the existence of vector fields on $\mathrm{M}$ called combings in [20]. We expect that with a suitable normalization the topological invariance of the formula up to a power of $\alpha(a)$ will follow as in [20]. In order to keep track of the power of $\alpha(a)$, we need further restrictions on decorations given by a choice of framing rather than a combing.

The invariance of $\#(M, T)$ on the decoration and triangulation absorbs all the general identities of balanced and semi-simple Hopf algebras.

In the interest of self-containedness, let us give an outline of the standard process of constructing a TQFT from a topologically invariant state sum [8]. First, we assign to any triangulated N-1 dimensional manifold $\mathrm{M}$ the vector space of formal linear combinations of all ways to label the simplices in the N-1 manifold as triangulated. For any two different triangulations of $\mathrm{M}$, we pick any triangulation of the N-manifold with boundary $M \times I$ which agrees with the two triangulations at the two ends. Next, we do the state sum on $M \times I$ for each choice of labels on the ends, summing over the interior. This gives us a set of matrix elements for a map between the two vector spaces associated to the two triangulations. Identifying under these maps gives us a real invariant vector space associated to M. It then follows from very general arguments that we obtain a TQFT.

\section{CHAPTER 2. THE DEFINITION OF HOPF CATEGORIES}

\section{Categories and Algebras}

We are now going to construct an analog of the structure of a Hopf algebra on a tensor category of a very special type.

To explain the idea of an analog of an algebraic structure on a category, let us consider first the category VECT of finite dimensional vector spaces. This category possesses two product operations, $\oplus$ and $\otimes$ and special objects $\mathbf{0}$ and $\mathbf{1}$ and the following isomorphisms:

$(A \otimes B) \otimes C \Leftrightarrow A \otimes(B \otimes C)$

$(A \oplus B) \oplus C \Leftrightarrow A \oplus(B \oplus C)$

$A \otimes(B \oplus C) \Leftrightarrow(A \otimes B) \oplus(A \otimes C)$

$A \oplus \mathbf{O} \Leftrightarrow A$

$A \otimes \mathbf{1} \Leftrightarrow A$

$A \oplus B \Leftrightarrow B \oplus A$

These isomorphisms satisfy certain equations, called coherence relations. This is completely parallel to the definition of a ring. We describe this by saying that VECT is a ring category. This structure is a categorical analog of a ring.

There is a natural extension of this category which we call Z-VECT, the category of $\mathrm{Z}$ graded vector spaces. Objects in this category have Z-graded dimensions, which we like to think of as finite Laurent polynomials in a formal variable. We will also use the category $\mathbf{Z} / \mathbf{n}$-VECT of cyclically graded vector spaces. 
What has happened is that equations in the ring correspond to isomorphisms in the category. There are then natural equations that the isomorphisms should satisfy, so that combining them in different orders to produce a larger isomorphism always gives consistent results. These were termed "coherence relations" by MacLane [23]. The coherence relations corresponding to the commutative and associative laws are the Stasheff hexagons and pentagons [14].

Thus, if we replace an algebraic structure by a categorical analog, we replace its axioms by a new set of more complex equations, which are its coherence relations. One of the fundamental ideas which we would like to emphasize is that if we start with an algebraic structure which can be used to construct a TQFT, than the coherence relations of a categorical analog of it are just right to construct a TQFT in one higher dimension. Our use of a Hopf category in 4D-TQFT is an application of this idea.

\section{Hopf Categories.}

Now let us describe the structure of a Hopf category.

Definition. A category is semisimple if each object is a direct sum of simple objects. A semisimple category is finitely generated if it has only finitely many inequivalent irreducible objects.

In this paper, we will have a special interest in finitely generated categories (in order to make all sums finite).

Definition. If $\mathbf{R}$ is a ring category, then $\mathbf{M}$ is a module category over $\mathbf{R}$ if $\mathbf{M}$ has an associative direct sum and we are given a functor $\mathbf{R} \times \mathbf{M} \rightarrow \mathbf{M}$ (denoted as multiplication) such that

$$
\begin{aligned}
& A_{1} \otimes\left(A_{2} \otimes R\right) \Leftrightarrow\left(A_{1} \otimes A_{2}\right) \otimes R \\
& \left(A_{1} \oplus A_{2}\right) \otimes R \Leftrightarrow\left(A_{1} \otimes R\right) \oplus\left(A_{2} \otimes R\right) \\
& A \otimes\left(R_{1} \oplus R_{2}\right) \Leftrightarrow\left(A \otimes R_{1}\right) \oplus\left(A \otimes R_{2}\right)
\end{aligned}
$$

and the isomorphisms satisfy the natural coherence relations.

The concept of module category is the categorical analog of the concept of a module.

Definition. An algebra category is a ring category which is also a VECT module such that the structure functors are module maps.

Note that the sets of morphisms between pairs of objects in a VECT module must possess a vector space structure, and that composition is bilinear. VECT modules are a categorical analog of vector spaces, so algebra categories are categorical analogs of algebras.

Now recall that the dual of a category has the same objects as the category, but with morphisms reversed. Similarly, the dual of any algebraic construction has diagrams corresponding to the first one, but with arrows reversed. The dual of a VECT module has a natural structure as a VECT module, including a natural direct sum.

Definition. A coalgebra category is a VECT module category whose dual is an algebra category.

This is equivalent to a VECT module category with a comultiplication functor $\Delta: \mathbf{A} \rightarrow \mathbf{A} \times \mathbf{A}$ satisfying the dual of the axioms of an algebra category (coassociativity, etc.).

Definition. A bialgebra category (without unit and counit) is a VECT module category which is both an algebra and a coalgebra category, together with a consistency natural transformation:

Figure 4

The associativity and coassociativity isomorphisms and the consistency

map $\alpha: \Delta(A) \otimes \Delta(B) \Leftrightarrow \Delta(A \otimes B)$ must satisfy the following four commuting cubes as coherence relations:

\section{Figure 5}

The first two of these are the Stasheff pentagon and the dual object for comultiplication. The latter two are new.

Finally, a Hopf category is a semisimple bialgebra category together with the categorical analogs of a unit, counit, Frobenius inner product and dual inner product. For brevity, we omit discussion of the corresponding coherence relations.

For each of the categories we have discussed above, we can form its Grothendieck ring by using the direct sum and tensor product to define additions and multiplications of formal linear combinations of objects in the category. One then recovers an algebra with structure analogous to the category. 


\section{The Structure Constants of A Hopf Category.}

The above definition is extremely abstract, and hence not that convenient to use in writing a numerical formula. We want to think of the associativity, coassociativity, and consistency isomorphisms of a Hopf category as determining some numbers as "matrix elements," which must satisfy certain axioms as a result of the four cubes. It will then transpire that these numbers can be naturally fitted into a triangulated 4-manifold, and that the properties they satisfy guarantee topological independence.

The idea of extracting a number from the associator of a tensor category is one which is familiar to physicists and chemists under the guise of $6 \mathrm{~J}$ symbols [16]. The classical setting for this idea is the representation category of the Lie group $\mathrm{SU}(2)$, which is used to understand the addition of spins in quantum mechanics.

The associator of a tensor category gives us a map

$A \otimes(B \otimes C) \Rightarrow(A \otimes B) \otimes C$.

In order to extract a minimal unit of information from this in a semisimple category, we let $\mathrm{A}$, $\mathrm{B}$, and $\mathrm{C}$ be irreducible objects, denoted $R_{1}, R_{2}, R_{3}$. We then pick irreducible subobjects $R_{12} \subset R_{1} \otimes R_{2}, R_{23} \subset$ $R_{2} \otimes R_{3} ; R_{123} \subset R_{1} \otimes R_{2} \otimes R_{3}$. These imply a choice of four tensor operators $\mu: R_{1} \otimes R_{2} \rightarrow R_{12}, \nu$ : $R_{12} \otimes R_{3} \rightarrow R_{123} ; \rho: R_{2} \otimes R_{3} \rightarrow R_{23}$, and $\sigma: R_{1} \otimes R_{23} \rightarrow R_{123}$.

In order to define the $6 \mathrm{~J}$ symbols in the simplest (although not the most invariant) way, let us assume that we have chosen bases for the space of tensor operators for each triple of simple objects in our category, and that our four choices are basis elements.

Now we can form $\nu \circ \mu$ and $\sigma \circ \rho$. These are elements of two different bases for the same space, namely the tensor operators from $R_{1} \otimes R_{2} \otimes R_{3}$ to $R_{123}$. The coefficient of the first in the expansion of the second in the first basis is a scalar, and is defined as a $6 \mathrm{~J}$ symbol.

We can graphically represent the $6 \mathrm{~J}$ symbol as follows:

Figure 6

The coherence cube implies that the associator can be combined to form more complex quantities called 3NJ symbols. There is a 3NJ symbol for every labelling of the triangulated boundary of a polyhedron, where edges are labeled with objects in the category and faces are labelled with tensor operators. The edges are directed, and the tensor operators, strictly speaking, depend on the directions, but can be reversed in a natural way using the duality of the category. Thus a triangle labelled

Figure 7

represents a tensor operator $R_{1} \otimes R_{2} \otimes R_{3} \rightarrow \mathbf{1}$; while a triangle

Figure 8

represents an operator $R_{1} \otimes R_{2} \rightarrow R_{3}{ }^{*}$. These can be naturally identified, however.

A $3 \mathrm{NJ}$ symbol can be evaluated by cutting up the interior of the polyhedron into tetrahedra, labelling internal edges with objects and internal faces with tensor operators, taking the product of the resulting $6 \mathrm{~J}$ symbols on the tetrahedra, then summing over the internal labellings. The result, by an application of the coherence cube, is independent of the choice of triangulation. This is a well known technique in the chemical literature for the case of $\mathrm{SU}(2)$, where it is called the graphical calculus [16].

Similarly, we have dual $6 J *$ and $3 N J *$ symbols corresponding to the coproduct and its coassociator.

The quantities produced by the graphical calculus are closely related to the construction of a 3D-TQFT by Turaev and Viro [8]. The construction first described by those two authors really does not require a braided tensor category, only a tensor category with a very weak condition on duals. One sufficient condition, perhaps not the weakest, is called "spherical" in [24].

Finally, the consistency isomorphism of a bialgebra category gives us a new set of numbers, which we call $3 \times 3 J$ symbols.

In order to specify a $3 \times 3 J$ symbol, we pick nine irreducible objects in the (finitely generated, semisimple) category $R_{i j}, \mathrm{i}, \mathrm{j}=1,2,3$. In addition, we pick "tensor operators" $\tau_{i}: R_{i 1} \otimes R_{i 2} \rightarrow R_{i 3}$ and "cotensor operators" $\kappa_{j}: R_{1 j} \square R_{2 j} \rightarrow \Delta\left(R_{3 j}\right)$, where we use the symbol $\square$ to denote an object in $A \times A$, rather than the tensor product of our bialgebra category $A$.

This gives us a number, because we can think of our data as giving us one map from $\Delta\left(R_{11} \otimes R_{22}\right)$ to $R_{33}$ and another from $\Delta R_{11} \otimes \Delta R_{22}$ to the same target. The coefficient of one in the expansion of the other in the first basis after composing with the compatibility isomorphism gives us the $3 \times 3 J$ symbol. In order to clarify this, let us introduce a graphical calculus, similar to the 3D-TQFT in [25]. 
Let us draw words in our bialgebra category as two dimensional triangular complexes. Let us place objects of our category on edges of faces (combinatorial 1-2 flags), and represent the comultiplication as a hinge where three triangles meet, and the multiplication as two edges of a triangle combining to produce a third.

In this language, we can draw the consistency isomorphism as follows;

Figure 9

This is closely related to an equation in the 3D-TQFT in [25]. Now on our new categorical level, it is a morphism, and should really be thought of as a 3D complex in 4 dimensions, a movie of a cup being squashed.

In this picture, the 3 tensor operators live on the 3 triangles, while the 3 cotensor operators inhabit the 3 hinges. This provides a topological picture for $3 \times 3 J$ symbols.

There is a natural family of combinations of $3 \times 3 J$ symbols, which we call $N-3 \times 3 J$ symbols. To specify one of these, we need $3 \mathrm{~N}$ irreducible objects in the category, $R_{i j} \mathrm{i}=1,2,3 ; \mathrm{j}=1,2 \ldots \mathrm{N}$; together with cotensor operators $\tau_{j}: R_{1 j} \otimes R_{2 j} \rightarrow R_{3 j}$ and $\kappa_{i}: R_{i 2} \square R_{i 3} \ldots R_{i N} \rightarrow \Delta^{N}\left(R_{i 1}\right)$.

We draw these as follows:

Figure 10

They are defined by breaking up the polygons into triangles, and summing over all labellings of the internal lines and triangles with triplets of irreducible objects joined by tensor operators and triplets of cotensor operators respectively, multiplying the resulting $3 \times 3 J$ symbols, and adding over labellings where the products of the cotensor operators on the triangles give the $\kappa_{i}$.

The coherence cube then assures us that the result is independent of the choice of triangulation of the polygon.

There is also a dual family of $N-3 \times 3 J *$ symbols, reversing the roles of tensor and cotensor operators in the above construction.

We refer to this property as the thickened associativity of $3 \times 3 J$ symbols.

\section{CHAPTER 3. THE TORNADO FORMULA}

The structural elements of a Hopf category we described above have a natural relationship with the topology of triangulated 4-dimensional manifolds.

Definition. Let $\mathrm{M}$ be a 4-manifold, and $\mathrm{T}$ be a triangulation of $\mathrm{M}$. Furthermore, let $\mathrm{A}$ be a finitely generated, semisimple Hopf category. A labelling of $(\mathrm{M}, \mathrm{T})$ with respect to $\mathrm{A}$ is an assignment of an irreducible object of A to each pair of an edge incident to a tetrahedron, together with an assignment of a basis element of the space of tensor operators to each pair of a face incident to a tetrahedron, joining the objects assigned to the edges of the triangle and the tetrahedron; and an assignment of a basis vector of the space of cotensor operators to each pair of an edge and a face around the loop of objects assigned to the loop of of tetrahedra containing the triangle.

Strictly speaking, the above definition is slightly ambiguous, since we need to specify which objects are in the domain and range of the tensor or cotensor operator. The dualities of the category allow us to identify different choices of this, for example $\operatorname{Hom}(A \otimes B, C) \Leftrightarrow \operatorname{Hom}\left(A, B^{*} \otimes C\right)$. We really need to orient our manifold and order the vertices, then use a convention to remove this ambiguity. It will then be necessary to prove that our invariant formula is independent of the choice of ordering. In the interest of brevity of notation and exposition, we will omit this wherever possible.

Let us denote by $\bigwedge(A, M, T)$ the (finite) set of labellings of $(\mathrm{M}, \mathrm{T})$ with respect to $\mathrm{A}$.

Now let us note that each $\lambda \in \Lambda(A, M, T)$ assigns to each tetrahedron $\mathrm{t}$ in the triangulation a $6 \mathrm{~J}$ symbol $6 J(t, \lambda)$, to each triangle f a $N-3 \times 3 J$ symbol $N-3 \times 3 J(f, \lambda)$, and to each edge e a $3 N J *$ symbol $3 N J *(e, \lambda)$.

In this interlocking set of assignments, each tensor and cotensor operator appears in two places, once corresponding to a triangle, and once on an edge or tetrahedron.

We can now form the following expression:

$\operatorname{TORN}(A, M, T)=$

$\sum_{\lambda \in \Lambda(A, M, T,)} \prod_{e} 3 N J *(e, \lambda) \prod_{f} N-3 \times 3 J(f . \lambda) \prod_{t} 6 J(t, \lambda)$

This expression, which we are calling the tornado, (partly because the configuration of labels around a triangle in 4-space reminds us of a vortex,), is a completely natural analog of the $3 \mathrm{D}$ formula. The sum seems 
to depend on our choices of bases for our spaces of tensor and cotensor operators. However, each operator actually appears twice, and if we include a convention using orientation as to exactly how it appears, the two appearances are in dual spaces. Thus, the dependence on the choice of basis actually cancels itself out.

THEOREM 1. The tornado formula is independent of the triangulation, and hence gives an invariant of 4-manifolds. If we take a relative version of the tornado formula on manifolds with boundary, it gives us a $4 \mathrm{D}-\mathrm{TQFT}$.

CONJECTURE 1. The tornado formula admits an extension to the case of a non-involutory Hopf category.

We believe that the extension will be analogous to the 3D case we discussed in Chapter 1 .

In this paper we will give the outline of a proof of theorem 1. A more complete treatment will appear in a later paper. We briefly discuss the role of conjecture 1 in the conclusions.

\section{Outline Of a Proof of Topological Invariance.}

Let us first outline a general strategy to prove that sums like the tornado are invariant, then show how the properties of a Hopf category allow us to carry out this method for the tornado.

A summation of the type of the tornado formula, where labels are attached someplace in a triangulation (for example, to combinatorial flags, or to simplices of certain dimensions), and some sort of combinations are computed (on other dimensional simplices), after which the labelling is summed over, is called a state sum.

Definition. We say a state sum has the blob property if the sum, when carried out on any triangulation of a ball, with the values of labels in the boundary of the ball fixed and the labels in the interior of the ball summed over, equals the value of the contributions from the boundary of the ball only.

The blob property can be thought of as a sort of Poincare lemma.

Lemma. A state sum with the blob property gives an invariant of closed manifolds. If applied to manifolds with boundary, it gives rise to a TQFT.

Outline of proof: Any two triangulations of an $\mathrm{n}$ dimensional manifold can be related by a series of elementary moves called "bistellar equivalences" due to Pachner [26]. The moves all consist in replacing one configuration with another with the same boundary. Thus, the blob lemma implies invariance under Pachner's moves. This means that a state sum with the blob property gives a value on a closed manifold which does not depend on the triangulation. There is a standard method for extending this to a TQFT by doing the state sum on triangulated manifolds with boundary [8], as we briefly explained in chapter 1 .

Now let us explain a general strategy for showing that a certain type of state sum has the blob property. By induction, it suffices to show that the join along some part of its boundary of a single simplex to a ball with some triangulation, if summed only over boundary contributions of the ball, and all contributions from the simplex, gives the same result as a sum over the boundary of the new larger ball resulting from the union.

Figure 11

In order to prove this equivalence, we can break it up into a series of two parts.

1.Rearrangement. We need to change the triangulation of the shared part of the boundary so it becomes identical to the other half of the boundary of the simplex.

2.Squashing. We need to be able to collapse down the "pillow" between one pair of overlapping simplices at a time.

The picture of the consistency isomorphism we drew above is an example of a squashing move. In the proof of the topological invariance of the 3D-TQFT constructed from a Hopf algebra in [25], this move plays essentially this role.

Now let us consider how to apply this strategy to the tornado formula. We need to be able to carry out two kinds of moves on our summation, rearrangements and squashings. Basically, the rearrangements follow from the coherence cubes of the multiplication; while the squashing move is one version of the coherency cube of associativity with the consistency isomorphism.

Figure 12

As in the three dimensional case, we expect that the invariance of the tornado will use all the identities of Hopf categories corresponding to semisimple Hopf algebras. Assuming conjecture 1, a similar relation should hold for balanced Hopf algebras as well. 


\section{Chapter 4. THE HOPF CATEGORY ASSOCIATED TO THE HOPF ALGEBRA $u_{q}^{+}\left(\mathfrak{s l}_{2}\right)$}

It is well-known that the quantum groups $U_{q}(\mathfrak{g})$ gives rise to a rich class of $3 D$-TQFTs, which can be viewed as combinatorial counterparts of the CSW path integrals. To construct a $3 D$-TQFT directly from a Hopf algebra, one only needs "half" of the quantum group, ie $U_{q}^{+}(\mathfrak{g})$. This approach presumably leads to the same CSW theory and avoids the preparatory work of constructing a modular tensor category. When $\mathfrak{g}=\mathfrak{s l}_{2}$, the Hopf algebra $U_{q}^{+}(\mathfrak{g})$ over $\mathbf{C}\left[q, q^{-1}\right]$ is generated by $e, q^{ \pm h}$ with the relations

$$
q^{h} q^{-h}=1, \quad q^{h} e=e q^{h+2} .
$$

The comultiplication is given by

$$
\Delta q^{h}=q^{h} \otimes q^{h}, \quad \Delta e=e \otimes 1+q^{h} \otimes e .
$$

In order to obtain a finite-dimensional Hopf algebra we have to consider the case when $q$ is a root of unity. We set $q=\exp (2 \pi i / 2 r)$ and we impose extra relations

$$
e^{r}=0, \quad\left(q^{h}\right)^{2 r}=1 .
$$

As a result, we obtain a finite dimensional Hopf algebra, which will be denoted $u_{q}^{+}\left(\mathfrak{s l}_{2}\right)$.

Before we proceed to the construction of a Hopf category associated to $u_{q}^{+}\left(\mathfrak{s l}_{2}\right)$ we will first discuss integral bases. For example, one can choose a basis $q^{n h} e^{m}, n \in \mathbf{Z}, m \in \mathbf{Z}_{+}$. Then the structure coefficients of multiplication and comultiplication belong to $\mathbf{Z}_{+}\left[q, q^{-1}\right]$. This basis would be sufficient for the purpose of this paper. However, we will prefer to consider another basis, which can also be extended to the full quantum group $U_{q}\left(\mathfrak{s l}_{2}\right)$.

Following Lusztig [27] we will introduce a modified Hopf algebra $\dot{U}_{q}^{+}\left(\mathfrak{s l}_{2}\right)$ with generators $e_{n}, 1_{n}, n \in \mathbf{Z}$, satisfying the relations

$$
1_{m} 1_{n}=\delta_{m, n} 1_{m}, \quad e_{m} 1_{n}=\delta_{m-1, n} e_{m}, \quad 1_{n} e_{m}=\delta_{m+1, n} e_{m} .
$$

Then if we define the comultiplication by

$$
\Delta 1_{n}=\sum_{n^{\prime}+n^{\prime \prime}=n} 1_{n^{\prime}} \otimes 1_{n^{\prime \prime}}, \Delta e_{n}=\sum_{n^{\prime}+n^{\prime \prime}=n}\left(e_{n^{\prime}} \otimes 1_{n^{\prime \prime}}+q^{n^{\prime}} 1_{n^{\prime}} \otimes e_{n^{\prime \prime}}\right)
$$

we obtain a Hopf algebra denoted $\dot{U}_{q}^{+}\left(\mathfrak{s l}_{2}\right)$. Setting

$$
e=\sum_{n \in \mathbf{Z}} e_{n}, \quad q^{ \pm h}=\sum_{n \in \mathbf{Z}} q^{ \pm n} 1_{n}
$$

in an appropriate completion of $\dot{U}_{q}^{+}\left(\mathfrak{s l}_{2}\right)$ one recovers the Hopf algebra $U_{q}^{+}\left(\mathfrak{s l}_{2}\right)$. We will consider the following basis in $\dot{U}_{q}^{+}\left(\mathfrak{s l}_{2}\right)$

$$
e_{m}^{(m)}=\frac{1}{[m] !} e_{n+m-1} e_{n+m-3} \ldots e_{n-m+1}, \quad n \in \mathbf{Z}, m \in \mathbf{Z}_{+}
$$

where $[m] !=[1][2] \ldots[m],[m]=\left(q^{m}-q^{-m}\right) /\left(q-q^{-1}\right)$. One has $e_{n}^{(0)}=1_{n}, e_{n}^{(1)}=e_{n}$. Then the multiplication and comultiplication in this basis admits the following form:

$$
e_{n}^{(m)} e_{n^{\prime}}^{\left(m^{\prime}\right)}=\left[\begin{array}{c}
m+m^{\prime} \\
m
\end{array}\right] \delta_{n-m^{\prime}, n^{\prime}+m} e_{n-m^{\prime}}^{m+m^{\prime}}
$$

$$
\triangle e_{n}^{(m)}=\sum_{\substack{m^{\prime}+m^{\prime \prime}=m \\ n^{\prime}+n^{\prime \prime}=n}} q^{n^{\prime} m^{\prime \prime}} e_{n^{\prime}}^{\left(m^{\prime}\right)} \otimes e_{n^{\prime \prime}}^{\left(m^{\prime \prime}\right)}
$$


Thus the structure coefficients of multiplication and comultiplication belong to $\mathbf{Z}_{+}\left[q, q^{-1}\right]$. Associativity of multiplication and comultiplication and their consistency yield, respectively the following identities in $\mathbf{Z}_{+}\left[q, q^{-1}\right]$

$$
\begin{gathered}
{\left[\begin{array}{c}
m+m^{\prime} \\
m
\end{array}\right]\left[\begin{array}{c}
m+m^{\prime}+m^{\prime \prime} \\
m+m^{\prime}
\end{array}\right]=\left[\begin{array}{c}
m+m^{\prime}+m^{\prime \prime} \\
m
\end{array}\right]\left[\begin{array}{c}
m^{\prime}+m^{\prime \prime} \\
m^{\prime}
\end{array}\right]} \\
q^{\left(n^{\prime}+n^{\prime \prime}\right) m^{\prime \prime \prime}} q^{n^{\prime} m^{\prime \prime}}=q^{n^{\prime}\left(m^{\prime \prime}+m^{\prime \prime \prime}\right)} q^{n^{\prime \prime} m^{\prime \prime \prime}} \\
{\left[\begin{array}{c}
m+m^{\prime} \\
m
\end{array}\right]=\sum_{m_{1}+m_{2}=m} q^{\tilde{m} m_{2}-\tilde{m}^{\prime} m_{1}}\left[\begin{array}{c}
\tilde{m} \\
m_{1}
\end{array}\right]\left[\begin{array}{c}
\tilde{m}^{\prime} \\
m_{2}
\end{array}\right]}
\end{gathered}
$$

, where

$$
m+m^{\prime}=\tilde{m}+\tilde{m}^{\prime}
$$

Again when $q=\exp (2 \pi i / 2 r)$ we can consider a finite dimensional Hopf algebra $\dot{u}_{q}^{+}\left(\mathfrak{s l}_{2}\right)$ with the basis $e_{n}^{(m)}, m=0,1, \ldots, r-1, n \in \mathbf{Z} / 2 r \mathbf{Z}$.

\section{The combinatorial construction of the Hopf category.}

To construct a Hopf category corresponding to $\dot{U}_{q}^{+}\left(\mathfrak{s l}_{2}\right)$ we replace the structure coefficients by graded vector spaces of the corresponding dimension. Let $V=\bigoplus_{k \in \mathbf{Z}} V_{k}$ be a finite dimensional graded space. We define its graded dimension by

$$
\operatorname{dim}_{q} V=\sum_{k \in \mathbf{Z}} q^{k} \operatorname{dim} V_{k}
$$

We also define a shift operator $T$ that shifts degree of elements by 1 and its inverse $T^{-1}$. Clearly one had $\operatorname{dim}_{q} T^{n} V=q^{n} \operatorname{dim}_{q} V, n \in \mathbf{Z}$. For each $n \in \mathbf{Z}$ we define a quantum $n$-dimensional vector space $V^{n}$ with a basis $\left\{v_{k}\right\}$ indexed by degree $k \in\{n-1, n-3, \ldots,-n+1\}$. We will identify $V^{1} \cong \mathbf{C}$. Then we have $\operatorname{dim}_{q} V^{n}=[n]$. Finally we denote by $\Lambda^{m} V^{n}$ the $m$-th exterior power of $V^{n}$, it has a natural basis $v_{k_{i}} \wedge \ldots \wedge v_{k_{m}}-n<k_{1}<\ldots<k_{m}<n$. One can easily show that

$$
\operatorname{dim}_{q} \Lambda^{m} V^{n}=\left[\begin{array}{c}
n \\
m
\end{array}\right]
$$

A complimentary choice of indices establishes an isomorphism $\Lambda^{m} V^{n} \simeq \Lambda^{n-m} V^{n}$. Now we are ready to replace the identities for the structure constants by the following linear isomorphisms

$$
\alpha: \Lambda^{m} V^{m+m^{\prime}} \otimes \Lambda^{m+m^{\prime}} V^{m+m^{\prime}+m^{\prime \prime}} \longrightarrow \Lambda^{m} V^{m+m^{\prime}+m^{\prime \prime}} \otimes \Lambda^{m^{\prime}} V^{m^{\prime}+m^{\prime \prime}}
$$

and

$$
\beta: T^{n^{\prime} m^{\prime \prime}} \mathbf{C} \otimes T^{\left(n^{\prime}+n^{\prime \prime}\right) m^{\prime \prime \prime}} \mathbf{C} \longrightarrow T^{n^{\prime}\left(m^{\prime \prime}+m^{\prime \prime \prime}\right)} \mathbf{C} \otimes T^{n^{\prime \prime} m^{\prime \prime \prime}} \mathbf{C}
$$

and

$$
\gamma: \Lambda^{m} V^{m+m^{\prime}} \longrightarrow \bigoplus_{m_{1}+m_{2}=m} T^{\tilde{m} m_{2}-\tilde{m}^{\prime} m_{1}} \Lambda^{m_{1}} V^{\tilde{m}} \otimes \Lambda^{m_{2}} V^{\tilde{m}^{\prime}}, m+m^{\prime}=\tilde{m}+\tilde{m}^{\prime}
$$

where $\alpha, \beta, \gamma$ admit a simple combinatorial description. One can break the basis of $V^{m+m^{\prime}+m^{\prime \prime}}$ into three subsets of order $m, m^{\prime}$ and $m^{\prime \prime}$, respectively, either by choosing first a subset of order $m+m^{\prime}$ and inside it a subset of order $m$ or by choosing first a subset of order $m^{\prime}+m^{\prime \prime}$ and inside it a subset of order $m^{\prime}$. The comparison between these two ways yields the isomorphism $\alpha$. The isomorphism $\beta$ is self-evident. To construct the isomorphism $\gamma$, we first break the basis of $V^{m+m^{\prime}}$ into two segments of order $\tilde{m}$ and $\tilde{m}^{\prime}$, correspondingly. Then any choice of $m$ basic elements in $V^{m+m^{\prime}}$ is automatically partitioned into two 
parts (say of orders $m_{1}$ and $m_{2}$ ) corresponding to the two segments. Since the degrees of elements in both segments are shifted in comparison with standard degrees of $V^{\tilde{m}}$ and $V^{\tilde{m}^{\prime}}$, we need an adjustment given by $T^{\tilde{m} m_{2}-\tilde{m}^{\prime} m_{1}}$.

We define a semisimple $\mathbf{Z}$-VECT module category $\dot{\mathfrak{U}}_{q}\left(\mathfrak{s l}_{2}\right)$ by indicating a set of simple objects $E_{n}^{(m)}, m \in$ $\mathbf{Z}_{+}, n \in \mathbf{Z}$, with $\operatorname{Hom}\left(E_{n}^{(m)}, E_{n^{\prime}}^{\left(m^{\prime}\right)}\right)=\mathbf{C}$ if $m=m^{\prime}, n=n^{\prime}$ and zero otherwise. We also define monoidal $\otimes$ and comonoidal $\triangle$ structure in $\dot{\mathfrak{U}}_{q}^{+}\left(\mathfrak{s l}_{2}\right)$ by

$$
\begin{aligned}
& E_{n}^{(m)} \square E_{n^{\prime}}^{\left(m^{\prime}\right)} \stackrel{\sim}{\Rightarrow}\left\{\begin{array}{l}
\wedge^{m} \vee^{m+m^{\prime}} \otimes E_{n+n^{\prime}}^{m+m^{\prime}}, n-m^{\prime}=n^{\prime}+n \\
0, \text { otherwise }
\end{array}\right.
\end{aligned}
$$

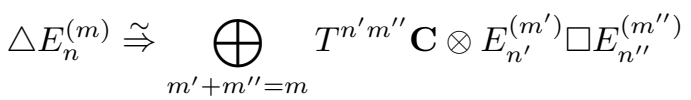

Then the linear isomorphism $\alpha$ induces an associativity isomorphism, $\beta$ induces a coassociativity isomorphism and $\gamma$ provides $\dot{\mathfrak{U}}_{q}^{+}\left(\mathfrak{s l}_{2}\right)$ with the structure of a bialgebra category. We obtain

Theorem 2. The semisimple $\mathbf{Z}$-VECT module category $\dot{\mathfrak{U}}_{q}^{+}\left(\mathfrak{s l}_{2}\right)$ provided with monoidal and comonoidal structures as above as well as associativity, coassociativity and consistency isomorphisms is a bialgebra category.

Proof. One needs to verify the pentagon relations for the associativity and coassociativity isomorphisms and their relations with the consistency isomorphism. This follows from the combinatorial descriptions of linear maps $\alpha, \beta, \gamma$. In fact, the pentagon for associativity follows from an analysis of the subdivision of the basis of $V^{m+m^{\prime}+m^{\prime \prime}+m^{\prime \prime \prime}}$ into subsets of order $m, m^{\prime}, m^{\prime \prime}$ and $m^{\prime \prime \prime}$ and the definition of $\alpha$. The relation between $\alpha$ and $\gamma$ follows from an analysis of a subdivision of the basis of $V^{m+m^{\prime}+m^{\prime \prime}}$ into parts of order $m, m^{\prime}$ and $m^{\prime \prime}$ relative to segments of order $\tilde{m}$, and $\tilde{m}^{\prime}$. Similarly, the relation between $\beta$ and $\gamma$ follows from an analysis of a subdivision of the basis of $V^{m+m^{\prime}}$ into parts of order $m$ and $m^{\prime}$ relative to segments of order $\tilde{m}, \tilde{m}^{\prime}$ and $\tilde{m}^{\prime \prime}$. Finally the pentagon for coassociativity follows from the relation between shifts of segments of $\tilde{m}, \tilde{m}^{\prime}, \tilde{m}^{\prime \prime}$ and $\tilde{m}^{\prime \prime \prime}$ in the basis of $V^{m}$.

When we pass to the root of unity case, we need to replace the category of graded vector spaces $\mathbf{Z}$-VECT by a category of $\mathbf{Z} / 2 r$-cyclicly graded vector spaces, then define a subquotient finitely generated category by a procedure reminiscent of the factorization of the subcategory of representations of a quantum group by the ideal subcategory of representations with zero quantum dimension. In addition, one needs to introduce categorical analogues to the bilinear forms in $u_{q}^{+}\left(\mathfrak{s l}_{2}\right)$.

One of our goals is the following:

Conjecture 2:. There exists a subquotient of the Hopf category associated to $U_{q}^{+}(\mathfrak{s l}(2))$ with the Grothendieck ring given by $\dot{u}_{q}^{+}\left(\mathfrak{s l}_{2}\right)$.

Clearly the combination of conjectures 1 and 2 should provide a combinatorial construction of four dimensional invariants for any $r \in \mathbf{Z}_{+}$. If true, they will yield a natural generalization of the celebrated $3 D$ invariants from quantum groups at roots of unity.

\section{CHAPTER 5. CONCLUSIONS}

In this paper we have outlined a new approach to constructing 4D-TQFT. Much work remains before we can say whether this construction can distinguish smooth structures, or has any relationship to DonaldsonFloer theory. We expect that in order to find examples of the tornado formula which are sensitive to smooth structure, it will be necessary to modify it to include cases of Hopf categories corresponding to nonsemisimple Hopf algebras, in particular the quantum groups.

Just as state sums associated to balanced Hopf categories are sensitive to framings of 3-manifolds, we expect the modified tornado formula to be sensitive to the choice of a bundle over the 4-manifold. Note that nontrivial bundles on 4-manifolds can be described via changes of framings over 3-manifolds which cut them. This would be similar to the dependence of DF theory on instanton number, which plays a crucial role in detecting smooth structures.

By analogy with the 3D case, we expect the relevant Hopf categories to contain grouplike objects, which can be used to insert instantons in the topological picture.

We synthesize our conjectures 1 and 2 into the following grand conjecture: 
Main Conjecture. The TQFT arising from the modification of the tornado formula appropriate to noninvolutive Hopf categories, when applied to the Hopf categories associated to quantum groups (described in chapter 4) is sensitive to smooth structure, and is related to Donaldson-Floer theory.

The combination of ideas we described in the introduction suggest more than one route towards construction of a 4D-TQFT. On the one hand, we could form the 2-category of module categories over our Hopf category [15]. This would form a very special tensor 2-category, which we could use to construct a $4 \mathrm{D}$ theory analogously to the $3 \mathrm{D}$ construction in [7].

On the other hand, we believe that our Hopf category itself appears as a category of representations of a more intricate algebraic structure, which we call a trialgebra. A trialgebra should have two multiplications and one comultiplication satisfying natural consistency relations.

The most interesting examples of trialgebras should have the Hopf categories associated to the quantum groups for categories of representations. There is strong evidence that the simple example of a Hopf category corresponding to the quantum group $U_{q}(s l(2))$ can be obtained from certain representations of the Virasoro algebra at $\mathrm{c}=1$, which are related to the $\mathrm{c}=1$ critical string.

The various algebraic constructions of low dimensional TQFTs fit together into a picture which we call the dimensional ladder.

Figure 13

A general programmatic discussion of the dimensional ladder will appear in [28]. 


\section{FIGURE CAPTIONS}

1. $2 \mathrm{D}$ topological move

2. The associative law

3. A decorated face

4. The consistency isomorphism

5. The coherence cubes of a Hopf category

6. A 6J symbol

7. An oriented triangle

8. A differently oriented triangle

9. Graphic expression of the consistency isomorphism

10. An $N-3 \times 3 J$ symbol

11. Inductive step for blob property

12. $4 \mathrm{D}$ squashing move $=$ coherence cube

13. The dimensional ladder 


\section{BIBLIOGRAPHY}

[1] M.F. Atiyah , Topological quantum field theories, Publ. Math. Inst. Hautes Etudes Sci., Paris, vol 68, 1989, p175-186

[2] E. Witten , Topological quantum field theory , Comm. Math. Phys., vol 117, 1988 p353-386

[3] G. Segal, Conformal Field Theory, in Proceedings of the International conference on Mathematical Physics, Swansea, 1988

[4] N. Reshetikhin and V.G. Turaev , Invariants of 3-manifold via link polynomials and quantum groups , Invent. math., vol 103, p547-597, 1991

[5] L. Crane, 2-d Physics and 3-d Topology, Commun. Math. Phys., vol135, p615-640, (1991)

[6] K. Walker, On Wittens 3 manifold invariants, unpublished

[7] G. Kuperberg, Involutory Hopf algebras and three-manifold invariants, Int. J. Math. vol2 p41-66, (1990)

[8] V. Turaev and O. Viro, State sum invariants of 3-manifolds and quantum $6 j$ symbols, Topology vol 31, p865-902, 1992

[9] M. Fukuma, S. Hosono, H. Kawai, Lattice topological field theory in two dimensions, Commun. Math. Phys. vol166, p157-175, (1994)

[10] A. Floer, An instanton invariant for 3-manifolds, Commun. Math. Phys. vol118, p215-240, 1988

[11] R. Dijkgraaf and E. Witten, Topological Gauge Theories and Group Cohomology, Commun. Math. Phys vol129, p393-429, 1990

[12] D. Yetter, Topological quantum field theories associated to finite groups and crossed G-sets, Journal of Knot Theory and its Ramifications volI, p1-20, 1992

[13] D. Freed and F. Quinn Chern Simons theory with finite gauge group, Commun. Math. Phys. to appear

[14] L. Crane and D. Yetter A Categorical Construction of 4D Topological Quantum Field Theories, in Quantum Topology, World Scientific 1993, L. Kauffman ed.

[15] M. Kapranov and V. Voevodsky, Braided Monoidal 2-Categories, 2-Vector Spaces and Zamolodchikov's Tetrahedra Equation, preprint

[16] A. P. Yutsis, I. B. Levinson and V. V. Vanagas, Mathematical Apparatus of the Theory of Angular Momentum (Israel Program for Scientific Translations, Jerusalem).

[17] G. Lusztig , Canonical bases arising from quantized enveloping algebras I, J. Amer. Math. Soc. vol 3, p447-498, 1990

[18] R.G. Larson and M.E. Sweedler, An associative orthogonal bilinear form for Hopf algebras, Amer. J. Math. vol 91, p75-94, 1969

[19] D.E. Radford, The order of the antipode of a finite dimensional Hopf algebra is finite, Amer. J. Math vol 98, p333-355, 1973

[20] G. Kuperberg A Definition of \# $(M, H)$ in the Non involutory Case, unpublished.

[21] R.G. Larson and D.E. Radford pages 187-195, Semisimple cosemisimple Hopf algebras , Amer. J. Math vol 109, 1987

[22] E. Witten, Quantum Field Theory and the Jones Polynomial, Commun. Math. Phys. 121 p351-399, 1989

[23] S. MacLane Categories for the working mathematician, Springer Verlag , 1988

[24] J. W. Barrett and B. W. Westbury, Spherical Categories, University of Nottingham preprint, 1994

[25] S. Chung, M. Fukama, and A. Shapere, , Structure of Topological Field Theories in Three Dimensions Int. J. Mod. Phys. A, p1305-1360, (1994)

[26] U. Pachner, P.L. Homeomorphic Manifolds are Equivalent by Elementary Shelling, Europ. J. Combinatorics vol 12, p129-145, 1991

[27] G. Lusztig , Introduction to Quantum Groups , Birkhauser , 1993

[28] L. Crane and I.B. Frenkel, A Representation Theoretic Approach to TQFT, to appear in the proceedings of the Gelfand seminar 